
of E-MRS Fall Meeting 2006, Warsaw, September 4-8, 2006

\title{
Structural Properties of Co and CoFe Electrodes Forming a Magnetic Tunnel Junction
}

\author{
L. Geadczuk*, P. Pankowski, W. Paszkowicz, \\ P. DŁużewski, M. Wójcik And P. PrzysŁupski \\ Institute of Physics, Polish Academy of Sciences \\ al. Lotników 32/46, 02-668 Warsaw, Poland
}

The $\mathrm{M}_{1} / \mathrm{MgO} / \mathrm{M}_{2}$ trilayer tunnel magnetoresistance systems are studied by means of X-ray diffraction, NMR, and transmission electron microscopy techniques. As $\mathrm{M}_{1}$ and $\mathrm{M}_{2}$ electrodes we used $\mathrm{Co}, \mathrm{Fe}$, and CoFe layers. The growth mechanism and structural quality of both electrodes and of the epitaxial $\mathrm{MgO}$ barrier forming the magnetic tunnel junctions are experimentally examined. It is shown that the crystallographic coherence of magnetic tunnel junctions across the $\mathrm{MgO}$ barrier is significantly disturbed by imperfect crystal structure of magnetic electrodes. The NMR results indicate a difference in short-range order between bottom and top electrodes.

PACS numbers: 81.15.Hi, 68.65.Ac, 61.10.Nz, 76.60.Lz, 68.37.Lp

\section{Introduction}

The tunnel magnetoresistance effect (TMR) is one of the most significant recent discoveries concerning spintronics. The TMR effect is observed in heterostructures composed of two magnetic electrodes separated by thin insulating layer. The variation of tunneling conductance is observed when the relative orientation of magnetization of the electrodes in magnetic tunnel junction (MTJ) is varied from parallel to antiparallel one. Recently MTJs with spin-polarized electron tunneling effect have been extensively studied for application in devices which could be potentially used in magnetic random access memories (MRAM) or magnetic read head sensors $[1,2]$.

The theoretically predicted TMR ratio, $R_{\mathrm{TMR}}$, for epitaxial $\mathrm{Fe}(001) / \mathrm{MgO}(001) / \mathrm{Fe}(001)$ is extremely high $\left(R_{\mathrm{TMR}}>10[3,4]\right)$, exceeding the earlier predicted value for epitaxial $\mathrm{Co} / \mathrm{MgO} / \mathrm{Co}$ MTJ with bcc $\mathrm{Co}(001)$ electrodes [5]. Following the theoretical predictions, several experiments have been carried out on epitaxial and polycrystalline $\mathrm{FM}_{1} / \mathrm{MgO}(001) / \mathrm{FM}_{2}$ junctions,

*corresponding author; e-mail: gladl@ifpan.edu.pl 
where $\mathrm{FM}_{1}$ and $\mathrm{FM}_{2}$ are ferromagnetic layers of MTJs [6-11]. The values of TMR effect obtained experimentally are lower than those theoretically predicted. Up to now, the TMR ratio of about $R_{\mathrm{TMR}}=4.1$ at room temperature has been reported [11]. Both experimental results and theoretical predictions indicate that the TMR effect strongly depends on the structural quality of the barrier and magnetic electrodes forming the tunnel junction. In this respect, the quality of the electrode/barrier interface seems to be of primary importance. To achieve the TMR effect close to the theoretical predictions limits, a possibility to precisely control the structural quality of the junction seems to be a very important issue. In this paper, we report our first results on MBE growth of epitaxial magnetic tunnel junctions involving the $\mathrm{MgO}$ barriers. The main goal of this work was to analyze the differences in structural ordering of the cobalt electrodes surrounding the $\mathrm{MgO}$ tunneling barrier.

\section{Experimental}

The $\mathrm{M}_{1} / \mathrm{MgO} / \mathrm{M}_{2}$ structures were grown by $\mathrm{MBE}$ on the (112̄0) $\mathrm{Al}_{2} \mathrm{O}_{3}$ substrate covered by epitaxial Mo buffer layer, $200 \AA$ thick. The growth process of the trilayer structure was carried out at room temperature. A $100 \AA$ thick $\mathrm{M}_{1}$ $\left(\mathrm{M}_{1}=\mathrm{Co}\right)$ layer was deposited as a first magnetic electrode of the junction. Then on the top of the first $\mathrm{M}_{1}$ layer, the insulating epitaxial $\mathrm{MgO}$ layer, $20 \AA$ thick, was deposited. The second electrode, $\mathrm{M}_{2}\left(\mathrm{M}_{2}=\mathrm{Co}\right.$ or FeCo $)$ was $100 \AA$ thick. An electron gun for $\mathrm{Co}$ and $\mathrm{MgO}$, and an effusion cell for Fe evaporation were used. The final trilayer structure was covered with a $100 \AA$ thick Mo layer to protect the structure against oxidation. Reflected high energy electron diffraction (RHEED) and Auger electron spectroscopy (AES) techniques were used for in situ structural and chemical analysis of fabricated heterostructures.

Crystal structure of the multilayer and local disorder of Co atoms in magnetic electrodes was determined by transmission electron microscope (TEM), $\mathrm{X}$-ray diffraction (XRD) and nuclear magnetic resonance (NMR) methods. X-ray diffraction data were collected with the help of an X'Pert MPD diffractometer (Panalytical) equipped with a $\mathrm{Cu} \mathrm{X}$-ray tube and a strip detector (for description see Ref. [12]). The spin echo NMR experiments were performed in zero fields using a broad band phase sensitive detection [13]. Spin echo amplitude was recorded in the frequency range of $150-310 \mathrm{MHz}$ at $4.2 \mathrm{~K}$ and the NMR spectra have been corrected for NMR enhancement factor and for usual $\omega^{2}$ spectrum intensity dependence. TEM observations were performed with the use of JEM 2000EX instrument (JEOL) operated at $200 \mathrm{kV}$ accelerating voltage. Cross-sectional specimens were prepared by mechanical polishing and dimpling terminated with ion milling.

\section{Results}

Figure 1 shows the transmission electron microscopy cross-section image of the $\mathrm{Co} / \mathrm{MgO} / \mathrm{Co}$ trilayer structure deposited on an $\mathrm{Al}_{2} \mathrm{O}_{3}$ substrate covered by 


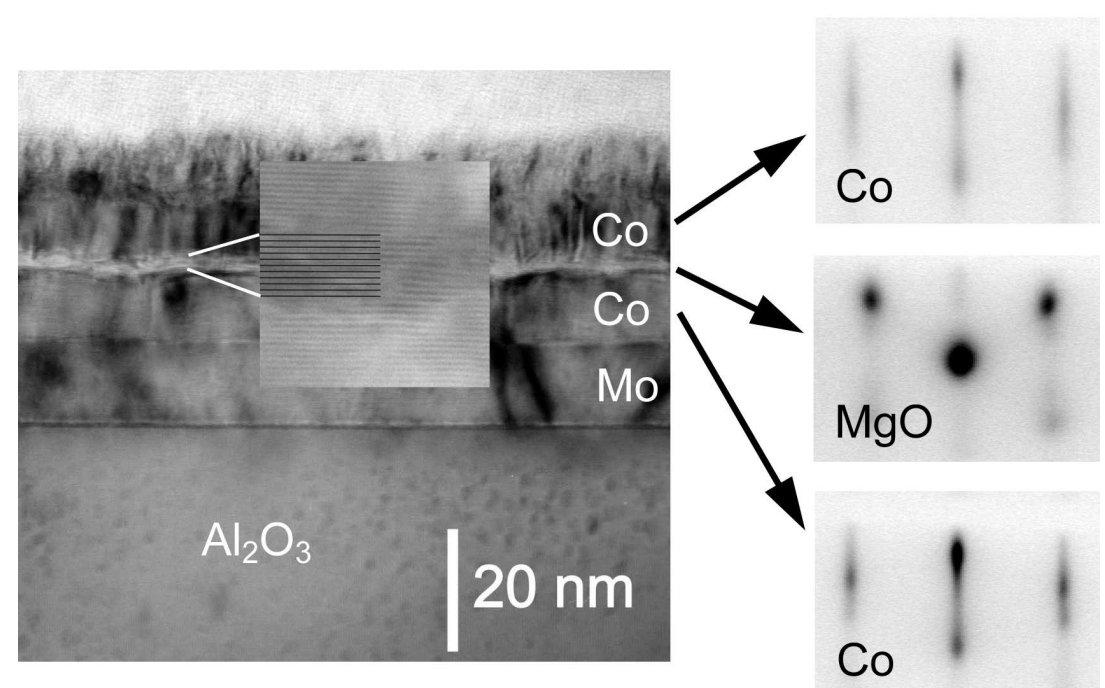

Fig. 1. TEM cross-section image of the $\mathrm{Co} / \mathrm{MgO} / \mathrm{Co}$ heterostructure and the RHEED patterns observed for $\mathrm{Co}, \mathrm{MgO}$, and Co layers during the growth.

a Mo buffer layer. A good quality of the Mo buffer structure and smooth interface with the Co bottom $\mathrm{M}_{1}$ layer are visible. The bottom Co layer revealed distortions originating from lattice mismatch between Mo and Co. $\mathrm{MgO}$ and Co interfaces exhibited a limited surface quality with roughness much smaller than the $\mathrm{MgO}$ barrier thickness. The $\mathrm{MgO}$ layer thickness determined from a lattice fringe image of the $\mathrm{Co} / \mathrm{MgO} /$ Co region (inset in Fig. 1) ranges from 10 to $12 \mathrm{ML}$. The top Co layer structure is much more distorted in respect of the bottom one. The electron diffraction measurements from selected area point out that this contact layer consists of a mixture of cubic and hexagonal Co phases. The RHEED patterns of the top and the bottom electrode and $\mathrm{MgO}$ barrier are also shown in Fig. 1. The patterns were recorded at different stages of deposition process of $\mathrm{Co} / \mathrm{MgO} / \mathrm{Co}$ structure. The RHEED patterns of the bottom Co layer indicate the epitaxial hcp layer growth in [111] direction and atomically flat surface. However, for the tunneling $\mathrm{MgO}$ barrier the pattern shows a 3D mode growth. The RHEED pattern from the upper Co layer indicates again epitaxial layer-by-layer growth. The $\mathrm{MgO}$ layer uniformly covers the cobalt surfaces and separates the two Co electrodes. For the trilayer structure with CoFe electrode, the observed RHEED pattern demonstrates a polycrystalline growth of CoFe layer.

$\mathrm{X}$-ray diffraction patterns obtained for the grown trilayers are presented in Fig. 2. All X-ray patterns show the peaks, which match the hcp and fcc structures of Co layers. However, the peaks from the particular crystal symmetry are poorly resolved. The dashed lines at the figure indicate the position of the peaks for the particular symmetry. The strong overlapping of Co hcp 002 and Fe 110 peaks hinders the phase analysis of the CoFe layer. 


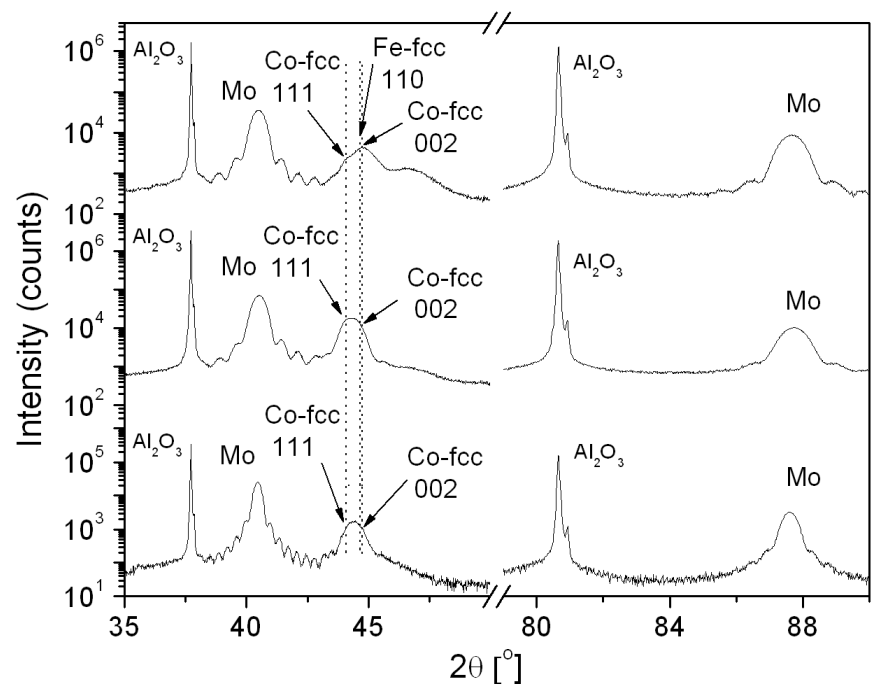

Fig. 2. XRD patterns of the $\mathrm{Co} / \mathrm{MgO}, \mathrm{Co} / \mathrm{MgO} / \mathrm{Co}$ and $\mathrm{Co} / \mathrm{MgO} / \mathrm{CoFe}$ trilayer structures deposited at Mo buffer layer at the $\mathrm{Al}_{2} \mathrm{O}_{3}$ substrate (from bottom to top). Peaks labeled by $\mathrm{Mo}$ and $\mathrm{Al}_{2} \mathrm{O}_{3}$ originate from the Mo buffer layer and substrate, respectively.

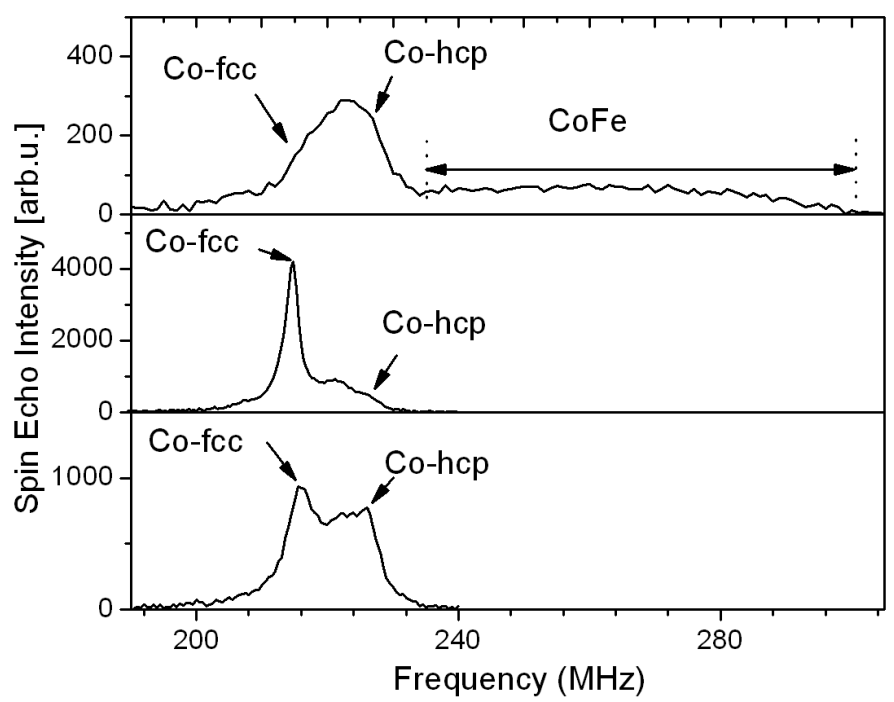

Fig. 3. ${ }^{59} \mathrm{Co} \mathrm{NMR}$ spectra at $4.2 \mathrm{~K}$ for the $\mathrm{Co} / \mathrm{MgO}, \mathrm{Co} / \mathrm{MgO} / \mathrm{Co}$ and $\mathrm{Co} / \mathrm{MgO} / \mathrm{CoFe}$ trilayer structures grown on $\mathrm{Al}_{2} \mathrm{O}_{3}$ substrate with Mo buffer layer (from bottom to top).

Figure 3 shows the ${ }^{59}$ Co NMR spectra of analyzed samples. The fcc and hcp Co phases in bulk samples give an NMR signal at slightly different frequencies, $217 \mathrm{MHz}$ for fcc Co and $226 \mathrm{MHz}$ for hcp Co — for magnetization in hexagonal $c$ plane. In samples with some fcc/hcp admixture, two additional NMR lines, at 
the intermediate frequencies, are observed and correspond to stacking faults. The presence of a strain in thin films can slightly alter these frequencies. In case of the studied trilayers the NMR spectra show that in all samples the Co layers are composed of both a good structural quality fcc phase and more or less faulted hcp phase occurring in different proportions. For the lower Co layer deposited on Mo buffer, the hcp stacking prevails, but stacking faults towards fcc structure and some amount of fcc phase are also present. A mixture of fcc and strongly faulted hcp phase becomes dominant in the upper Co layer deposited on $\mathrm{MgO}$ interlayer. A very broad feature is observed for the sample with CoFe layer, revealing a random intermixing of Co and Fe without any clear tendency of ordering towards $B 2$ structure.

\section{Discussion}

There are large discrepancies between theoretical and experimental TMR effect values of $\mathrm{M}_{1} / \mathrm{MgO} / \mathrm{M}_{2}$ tunnel junctions. It is known that the conductance of the junction depends critically on the symmetry of involved electronic states [14].

The results presented in this paper show that the spread of the value of TMR ratio could be strongly related to the structural quality of the layers of the junction and the structural quality of the $\mathrm{MgO} /$ electrode interfaces. The initial growth conditions of the two magnetic layers are significantly different, which influences the quality of their crystal structure (namely, the lower Co electrode grew on Mo buffer layer, whereas the upper $\mathrm{Co}$ and $\mathrm{CoFe}$ electrodes grew on the $\mathrm{MgO}$ interlayer). Comparison of XRD spectrum obtained for the sample containing one Co layer only to this recorded from the structure built from two Co layers around $\mathrm{MgO}$ layer did not allow to discriminate in XRD spectrum between the peaks from bottom Co and upper Co layer. This suggests that the long-range order in the electrodes on both sides of $\mathrm{MgO}$ layer is similar. However, the short-range order revealed by NMR is clearly different for the bottom Co and the upper Co electrode. A strong preference for hcp stacking in the lower Co layer was found. In case of CoFe electrode the picture emerging from XRD spectrum is not clear because of the close position of Fe 110 and hcp Co 002 peaks. Instead, a significant difference in Co atom arrangement in the lower and the upper magnetic layer is visible in the NMR spectrum. The upper electrode in this case is characterized by the short-range order which corresponds to bcc symmetry characteristic of the alloys with composition close to $\mathrm{Co}_{50} \mathrm{Fe}_{50}$. The uniformity of the electronic structure is additionally interrupted by the difference in phase proportion. There are significant differences in fcc and hcp phase proportion, and in the density of stacking faults in both Co electrodes. The different structure of the two cobalt layers introduces a difference in the two $\mathrm{Co} / \mathrm{MgO}$ and $\mathrm{MgO} / \mathrm{Co}$ interfaces, which implies a difference in the electronic structure at two sides of the tunneling barrier. The short-range atomic disorder in the electrodes breaks the symmetry of the $\mathrm{MgO}$ (001) barrier which could significantly disturb the structural coherence of 
the junction. Further investigation is needed to correlate the local disordering of Co electrodes with the transport properties of the junction.

\section{Conclusions}

The $\mathrm{Co} / \mathrm{MgO} / \mathrm{M}(\mathrm{M}=\mathrm{Co}$ or $\mathrm{CoFe})$ structures on $\mathrm{Al}_{2} \mathrm{O}_{3}$ substrate and Mo buffer layer were prepared by MBE method. The experimental results show that the crystallographic coherence of MTJs across the MgO barrier is significantly affected by imperfect crystal structure of magnetic electrodes. The bottom Co electrode displays the growth of the hcp phase which is structurally imperfect a clear tendency to form stacking faults towards the fcc phase is present. The fcc phase becomes dominant in upper Co layer deposited on $\mathrm{MgO}$ interlayer. The different arrangement of Co atoms in both magnetic layers can have significant influence on TMR value obtained for MTJs.

\section{Acknowledgments}

The research was partially supported by the Network "New materials and sensors for optoelectronics, information technology, energetics and medicine".

\section{References}

[1] J.S. Moodera, L.R. Kinder, T.M. Wong, R. Meservey, Phys. Rev. Lett. 74, 3273 (1995).

[2] T. Miyazaki, N. Tezuka, J. Magn. Magn. Mater. 139, L231 (1995).

[3] W.H. Butler, X.-G. Zhang, T.C. Schulthess, Phys. Rev. 63, 054416 (2001).

[4] J. Mathon, A. Umerski, Phys. Rev. B 63, 220403 (2001).

[5] X.-G. Zhang, W.H. Butler, Phys. Rev. B 70, 172407 (2004).

[6] C. Tusche, H.L. Meyerheim, N. Jedrecy, G. Renaud, A. Ernst, J. Henk, P. Bruno, J. Kirschner, Phys. Rev. Lett. 95, 176101 (2005).

[7] T. Moriyama, C. Ni, W.G. Wang, X. Zhang, J.Q. Xiao, Appl. Phys. Lett. 88, 222503 (2006).

[8] M. Mizuguchi, Y. Hamada, R. Matsumoto, S. Nishioka, H. Maehara, K. Tsunekawa, D.D. Djayaprawira, N. Watanabe, T. Nagahama, A. Fukushima, H. Kubota, S. Yuasa, M. Shiraishi, Y. Suzuki, J. Appl. Phys. 99, 08 T309 (2006).

[9] S. Yuasa, T. Nagahama, A. Fukushima, Y. Suzuki, K. Ando, Nature Mater. 3, 868 (2004).

[10] S.S.P. Parkin, C. Kaiser, A. Panchula, P.M. Rice, B. Hughes, M. Samant, S.-H. Yang, Nature Mater. 3, 862 (2004).

[11] S. Yuasa, A. Fukushima, H. Kubota, Y. Suzuki, K. Ando, Appl. Phys. Lett. 89, 042505 (2006).

[12] W. Paszkowicz, Nucl. Instrum. Methods Phys. Res. A 551, 162 (2005).

[13] P. Panissod, J.P. Jay, C. Mény, M. Wójcik, E. Jędryka, Hyperfine Interact. 97, 75 (1996).

[14] X.-G. Zhang, W.H. Butler, A. Bandyopadhyay, Phys. Rev. B 68, 092402 (2003). 